\title{
Engineering Novel S-Glycosidase Activity into Extremo-Adapted $\beta$-Glucosidase by Rational Design
}

\author{
Nouarh Almulhim ${ }^{1}$, Nicholas R. Moody ${ }^{1}$ and Francesca Paradisi ${ }^{1,2 *}$ \\ ${ }^{1}$ University of Nottingham, School of Chemistry, University Park, Nottingham NG7 2RD, United Kingdom \\ ${ }^{2}$ Department of Chemistry and Biochemistry University of Bern, Freiestrasse 3, 3012, Bern, Switzerland. \\ *Corresponding author. Department of Chemistry and Biochemistry University of Bern, Freiestrasse 3, 3012, \\ Bern, Switzerland. Tel: +41316314002 E-mail : francesca.paradisi@dcb.unibe.ch. \\ Nouarh Almulhim ORCID iD: 0000-0002-0845-4918 \\ Nicholas R. Moody ORCID iD: 0000-0002-0662-686X \\ Francesca Paradisi ORCID iD: 0000-0003-1938-262X
}


ABSTRACT

The breakdown of sulphur glycosidic bonds in thioglycosides can produce isothiocyanate, a chemoprotective agent linked to the prevention of cancers, however only a handful of enzymes have been identified that are known to catalyse this reaction. Structural studies of the myrosinase enzyme, which is capable of hydrolysing the thioglycosidic bond, has identified residues that may play important roles in sulphur bond specific activity. Using rational design, two extremo-adapted $\beta$-glycosidases from the species Thermus nonproteolyticus (TnoGH1) and Halothermothrix orenii (Hor $\mathrm{GH1}$ ) were engineered towards thioglycoside substrates. Twelve variants, six for TnoGH1and six for $\operatorname{Hor} \mathrm{GH} 1$, were assayed for activity. Remarkable enhancement of the specificity $\left(k_{\mathrm{cat}} / K_{\mathrm{M}}\right)$ of TnoGH1 and Hor GH1 towards $\beta$-thioglycoside was observed in the single mutants TnoGH1-V287R $\left(2500 \mathrm{M}^{-1} \mathrm{~s}^{-}\right.$ $\left.{ }^{1}\right)$ and $\operatorname{Hor} \mathrm{GH} 1-\mathrm{M} 229 \mathrm{R},\left(13260 \mathrm{M}^{-1} \mathrm{~s}^{-1}\right)$ which showed a 3-fold increase with no loss in turnover rate when compared to the wild type enzymes . Thus, the role of arginine is key to induce $\beta$-thioglycosidase activity. Thorough kinetic investigation of the different mutants has shed light on the mechanism of $\beta$-glycosidases when acting on the native substrate.

\section{KEY POINTS}

15 Key residues were identified in the active site of Brevicoryne brassicae myrosinase.

16 Rationally designed mutations were introduced into two extremo-adapted $\beta$-glycosidases.

$17 \beta$-glycosidases mutants exhibited improved activity against thioglycosidic bonds.

18 The mutation to arginine in the active site yielded the best variant.

\section{KEYWORDS}

20 Enzyme engineering, Site directed mutagenesis, glycoside hydrolase, Thermus nonproteolyticus, Halothermothrix orenii, myrosinase

\section{INTRODUCTION}

The glycoside hydrolase family 1 of enzymes (GH1; EC 3.2.1.21) is characterised by the ability to catalyse the hydrolysis of glycoside linkages in a variety of sugars ( $\beta$-glucosides) (Davies and Henrissat 1995; Park et al. 2017). In terms of structure, it has been demonstrated that $\mathrm{GH} 1$ are $(\beta / \alpha)_{8}$ barrel folded enzymes, which consist of eight twisted, parallel $\beta$-strands, located in the internal part of the protein, surrounded by eight $\alpha$-helixes in the external part. The C-terminal (in the $\beta$ strand 8 ) of all known $(\beta / \alpha)_{8}$ barrel proteins hosts the active site residues within the $\beta \rightarrow \alpha$ loop (Henn-Sax et al. 2001; Silverman et al. 2001). The hydrolytic ability of GH1 is dependent on two critical glutamic acid residues (Fig. 1), E164 (Thermus nonproteolyticus glycoside hydrolase, TnoGH1 numbering), located in the $\mathrm{T}^{161} \mathrm{LNEP}^{165}$ motif ( $\beta$-strands 4 ), is the acid catalyst, and a second one, E338, in the ${ }^{336}$ TENG $^{340}$ motif ( $\beta$-strands 7) is the nucleophile (Wang et al. 2003) (Fig. 1a). E164 plays an important role in the formation of the intermediate (enzyme-saccharide) of classical glycosidases as an activator of the glycosidic oxygen.

Thioglycosides are among the most stable glycosidic molecules. In these structures, the glycoside is bridged to the aglycon moiety by a sulphur bond. The breakdown of S- glycosidic bonds in glucosinolates (GSL) can release molecules with activity against pests and herbivores (part of the plant defence mechanism) and 
Myrosinases (EC 3.2.3.147) are unique members of the GH1 family able to hydrolyse thioglycosides. Unlike $\beta$-glycosidases which are ubiquitous, myrosinases have been identified only in a handful of species such as Sinapis alba (Burmeister et al. 1997), Brevicoryne brassicae (Jones et al. 2002), Verticillium longisporum (Witzel et al. 2015), Arabidopsis thaliana, and Brassica napus (Nong et al. 2010). The active site of Sinapis alba myrosinase ( $\mathrm{SaMYR}$ ), a plant species, differs from that of classical $\beta$-glycosidases, as it lacks the catalytic glutamic acid residue in the $\mathrm{T}^{184} \mathrm{INQL}^{188}$ motif (equivalent to $\mathrm{T}^{161} \mathrm{LNEP}^{165}$ in TnoGH1) (Bourderioux et al. 2005), while it maintains the second one (E409 in motif $\mathrm{T}^{408} \mathrm{ENG}^{411}$ ) (Burmeister et al. 1997). (Fig. 2a). In addition, $\mathrm{SaMYR}$ requires ascorbic acid as a cofactor to catalyses the hydrolysis of thioglycosidic substrates.

A myrosinase from the cabbage aphid Brevicoryne brassicae (BbMYR) on the other hand, relies on the typical 48 catalytic acid/base system found in $\beta$-glycosidases (E167 and E374) (Fig. 2b) and it more closely aligns with classic $\beta$-glycosidases than $S a$ MYR in term of structure and amino acid residues present in the active site. $B b \mathrm{MYR}$ has however unique structural features, not observed in either $\beta$-glycosidases or other myrosinases. K173 and R312 (Fig. 3) play a critical role in the hydrolysis of GSL, as they are directly involved in its recognition (Jones et al. 2002). Y180 may also play a role due to its proximity to the thioglycosidic linkage in the substrate (Husebye et al. 2005). Figure S1 shows full sequence alignment of Thermus nonproteolyticus glycoside hydrolase (TnoGH1), Halothermothrix orenii (Hor GH1), cabbage aphid Brevicoryne brassicae (BbMYR), and Sinapis alba myrosinase ( $\mathrm{SaMYR}$ ).

A thermophilic GH1 from the extremophilic bacterium Thermus nonproteolyticus (TnoGH1) and a halotolerant $\mathrm{GH} 1$ isolated from Halothermothrix orenii (Hor $\mathrm{GH} 1)$ have been previously described in the literature (He et al. 2001; Kori et al. 2011). Enzymes from extremophile organisms have significantly higher tolerance than the mesophilic counterparts to temperature and/or $\mathrm{pHs}$, making them attractive for industrial applications (Yin et al. 2015), however, no known examples have been reported of extremophilic GH1 with myrosinase activity. Engineering extremo-adapted GH1 to broaden their substrate scope towards $\beta$-thioglycosidase activity could significantly increase their potential applications in an industrial setting.

Here we report how rational design aided the introduction of amino acid mutations by mapping the $B b \mathrm{MYR}$ active site onto the extremophilic TnoGH1 enzyme first, and then onto Hor $\mathrm{GH}$ to further confirm the key role played by selected residues in the recognition and hydrolysis of thioglycosides. In TnoGH1, the mutations L171K, V287R, and H178Y were introduced as single and double mutants (generating 3 additional variants with all possible permutations). The equivalent single and double mutants at positions E173K, M299R, and H180Y were then introduced into $\operatorname{Hor} \mathrm{GH} 1$. The kinetic properties of all variants and wild type enzymes with test substrates $\beta$ D-thioglucopyranoside (pNT-Glc) and 4-nitrophenyl- $\beta$-D-glucopyranoside (pNP-Glc) have been carried out and the role of arginine (TnoGH1-V287R and HorGH1-M299R) has been found to be pivotal as a marked improvement of activity towards the $\beta$-thioglucoside substrate has been observed in both mutant proteins. These are the first examples of extremophilic GH1s in which myrosinase activity has been introduced. 


\section{MATERIALS AND METHODS}

\section{Reagents and bacterial strains}

Substrates 4-nitrophenyl $\beta$-D-thioglucopyranoside (pNT-Glc) and 4-nitrophenyl- $\beta$-D-glucopyranoside (pNPGlc) were purchased from Carbosynth. Escherichia coli bacterial strains and QuikChange II Site-Directed Mutagenesis Kit were from Agilent Technologies. The plasmid miniprep kit was from Macherey-Nagel. Growth media and assay components were procured from Fisher Scientific. All other chemicals were purchased from Sigma.

\section{DNA preparation and site-directed mutagenesis}

Mutations were introduced using the QuikChange Site-Directed Mutagenesis kit. pCH93b hosting the ds-DNA of TnoGH1 (GenBank accession number AF225213) and pET45b hosting the ds-DNA of Hor GH1(GenBank accession number WP_012636460) respectively were used as templates in the PCR reactions (Heckmann 2017). The mutagenic primers were designed using QuikChange Primer Design Program (www.agilent.com/genomics/qcpd). Primers are summarised in Table S1.

\section{Protein expression and purification}

For protein expression, BL21(DE3) E. coli strain was used. Chemically competent E. coli cells were transformed with each plasmid. A $300 \mathrm{~mL}$ LB flask was inoculated with starter culture and grown at $37^{\circ} \mathrm{C}(200$ $\mathrm{rpm})$ to an $\mathrm{OD}_{600}$ of $\sim 0.6$, prior to induction with $1 \mathrm{mM}$ IPTG. Induced cultures were then incubated at $37{ }^{\circ} \mathrm{C}$ overnight. Cells were harvested at $3,500 \times \mathrm{g}, 4^{\circ} \mathrm{C}, 20 \mathrm{~min}$. The pellet was resuspended in loading buffer $(50 \mathrm{mM}$ HEPES pH 7.5, $150 \mathrm{mM} \mathrm{NaCl}, 10 \mathrm{mM}$ imidazole) and lysed by sonication for 20 minutes on ice ( $1 \mathrm{~min}$ on, 30 sec off; 20 cycles). The soluble fraction was decanted following centrifugation at 22,800 $\times g, 4{ }^{\circ} \mathrm{C}$ for 1 hour, and filtered with a $0.45 \mu \mathrm{m}$ filter. Filtered supernatant was loaded onto a $1 \mathrm{~mL}$ HisTrap FF crude ${ }^{\circledR}$ column, using an AKTA $^{\text {TM }}$ Start. The column was washed with eight column volumes of loading buffer, followed by fifteen column volumes of loading buffer with $10 \%$ elution buffer (50 mM HEPES pH 7.5, 150 mM NaCl, $300 \quad \mathrm{mM}$ imidazole). The protein was then eluted with eight column volumes of $100 \%$ elution buffer. Pure fractions were pooled and dialysed for 20 hours at room temperature with dialysis buffer (50 mM HEPES pH 7.5, $150 \mathrm{mM}$ $\mathrm{NaCl})$, with one buffer exchange after $2 \mathrm{~h}$.

\section{Enzyme quantification}

100 The concentration of the purified enzymes was determined by absorbance at $280 \mathrm{~nm}$. The extinction coefficient $\varepsilon$ was estimated using the EXPasy ProtParam tool (Gasteiger et al. 2005) (Table S2). Proteins were analysed with a $12 \%$ SDS PAGE, by staining with InstantBlue (Fig. S2).

\section{Kinetics assays}

104 Enzyme activity was measured spectroscopically in triplicate by monitoring the change in absorbance at 420 $105 \mathrm{~nm}$ of the p-nitrophenol or p-nitrothiophenol. The extinction coefficients for the products was determined using a calibration curve (Fig. S3).

Assays were conducted in $200 \mu \mathrm{L}$ at $50^{\circ} \mathrm{C}$ for $T n o \mathrm{GH} 1$ and at $25^{\circ} \mathrm{C}$ for $H o r \mathrm{GH} 1$. A typical reaction mixture contained $100 \mathrm{mM}$ HEPES buffer pH 7.5, $500 \mathrm{mM}$ sodium chloride. pNT-Glc was dissolved in 30\% DMSO, the 
$110 \quad 0.45 \mu \mathrm{m}$ filter prior to use. Assays were initiated with the addition of enzyme. Primary non-linear regression plots

111 are described in Fig S5, S6, S7 and S8.

\section{Data analysis}

113 In silico modelling of crystal structures was performed using the UCSF Chimera software (Pettersen et al. 114 2004). Sequence alignments were determined with ENDscript server software (Robert and Gouet 2014).

115 Similarities and identities, including homology modelling, between sequences were calculated using EMBOSS

116 software (Rice et al. 2000). Kinetic parameters were evaluated by nonlinear regression analysis in Igor Pro 117 (Babonneau 2010). Bar graphs were produced in GraphPad Prism (Swift 1997). The primary plots were analysed 118 using equation 1. For consensus analysis of amino acids, $\beta$-glycosidase sequences were taken from the Pfam 119 protein family's database (El-Gebail et al. 2019), 4000 sequences of representative proteome were used for 120 amino acid analysis. The representative proteome at $15 \%$ comembership threshold, as defined by Pfam which is 121 an even sampling of the sequences of the glycosyl hydrolase family PF00232 (Chen et al. 2011), was aligned with 122 the wild type sequences of the $\beta$-glycosidase used in this study with the ClustalW tool with the MEGA X software 123 (Kumar et al. 2018). Sequence logo analysis was performed with WebLogo (Crooks et al. 2004).

$$
\frac{v_{i}}{[E]_{T}}=\frac{k_{c a t}^{a p p}[S]}{K_{M}^{a p p}+[S]} \quad \text { (Equation 1) }
$$

\section{RESULTS}

\section{Computational study of TnoGH1, HorGH1, BbMYR, and SaMYR}

A comparative study between the amino acid sequences of the four glycosyl hydrolases (TnoGH1, Hor GH1, $B b \mathrm{MYR}$, and SaMYR) was carried out (Fig. S1). Both TnoGH1 and Hor GH1, as expected, present a greater sequence similarity to the $B b$ MYR than $S a$ MYR. TnoGH1 and BbMYR show a 53.6\% similarity ( $34.3 \%$ identity), greater than that observed between TnoGH1 and $\mathrm{SaMYR}$ (46.3\% similarity and 30.0\% identity). Hor GH1 has a $58.1 \%$ similarity (37.2 \% identity) with $B b$ MYR and a 51.9\% (33.1\% identity) with $S a M Y R$. The active site is highly conserved across all enzymes.

\section{TnoGH1 and HorGH1 mutants construction}

133 Three residues in the amino acid sequence of BbMYR - K173, R312, and Y180, were identified to be important 134 in the hydrolysis of thioglucosides (Jones et al. 2002; Husebye et al. 2005). The sequence alignment between 135 BbMYR and TnoGH1 (Fig. 3) reveals that TnoGH1 presents a leucine, a valine, and a histidine at the equivalent 136 positions (L171, V287, and H178 - TnoGH1 numbering). Likewise, in HorGH1 (Fig. 3) three residues (E173, 137 M299 and H180) were selected to be replaced by K, R, and Y, respectively. Three single mutants were generated 138 in both the thermotolerant enzyme TnoGH1 (L171K, V287R, and H178Y) and the halotolerant Hor GH1 (E173K, 139 M299R, and H180Y). Double mutant permutations were then created (L171K/V287R, L171K/H178Y, and $140 \mathrm{~V} 287 \mathrm{R} / \mathrm{H} 178 \mathrm{Y}$ in TnoGH1, and E173K/ M299R, E173K/H180Y, and M299R/H180Y in HorGH1) to elucidate 141 any synergistic effect among these amino acids. All mutants were expressed and purified (Fig. S2).

143 BbMYR activity against sinigrin (Fig. 4), the native substrate, was reported with $k_{\text {cat }}=36 \mathrm{~s}^{-1}, K_{\mathrm{M}}=0.41 \mathrm{mM}$ 144 (Pontoppidan et al. 2001). Both TnoGH1 and HorGH1 exhibited some activity against pNT-Glc, used here as 
145 substrate mimic. All determined kinetic parameters are summarised in Table 1 and Table 2. For TnoGH1, $k_{\text {cat }}$ of $1461.34 \mathrm{~s}^{-1}, K_{\mathrm{M}}$ of $1.43 \mathrm{mM}$ and $k_{\text {cat }} / K_{\mathrm{M}} 940 \mathrm{M}^{-1} \mathrm{~s}^{-1}$ were observed (Fig. S4). For Hor GH1, $k$ cat of $37.20 \mathrm{~s}^{-1}, K_{\mathrm{M}}$ of $1474.10 \mathrm{mM}$ and $k_{\text {cat }} / K_{\mathrm{M}} 9083 \mathrm{M}^{-1} \mathrm{~s}^{-1}$ were measured (Fig. S4), identifying the latter as the better catalyst towards 148 pNT-Glc $\left(k_{\text {cat }}\right.$ of $\sim 28$-fold higher than TnoGH1).

\section{TnoGH1 and HorGH1 single mutant kinetic studies (pNT-Glc)}

150 When compared to the wild type, the TnoGH1-V287R single mutant shows the greatest increase in specificity, $151 k_{\mathrm{cat}} / K_{\mathrm{M}}$, and a retention of turnover rate, $k_{\mathrm{cat}}$ (Table 1) with pNT-Glc.

152 The mutant TnoGH1-L171K shows a near retention in specificity $\left(1200 \mathrm{M}^{-1} \mathrm{~s}^{-1}\right)$ and turnover rate $\left(1.10 \mathrm{~s}^{-1}\right)$, 153 while TnoGH1-H187Y shows a 3-fold loss in specificity $\left(300 \mathrm{M}^{-1} \mathrm{~s}^{-1}\right)$ and a 10 -fold loss in turnover rate $\left(0.15 \mathrm{~s}^{-}\right.$ $\left.1544^{1}\right)$

TnoGH1-V287R shows an improvement in specificity with no loss of turnover rate. The combined improvement in $k_{\mathrm{cat}} / K_{\mathrm{M}}$ and retention of $k_{\mathrm{cat}}$ identifies TnoGH1-V287R as the best mutant for practical application. The kinetic parameters of TnoGH1 are summarised in Table 1.

A similar pattern is observed in the $\operatorname{Hor} \mathrm{GH} 1$ mutants. $\operatorname{Hor} \mathrm{GH} 1-\mathrm{M} 299 \mathrm{R}$ shows over $30 \%$ increase in specificity and a retention of turnover number (Table 2). While little change is observed in TnoGH1-L171K, Hor GH1-E173K shows a 3-folld loss in specificity and a 2-fold loss in turnover rate. The Hor GH1-H180Y mutant again shows a 3 -fold decrease in specificity and a 4-fold decrease in turnover compared to the wild type, similar to the change observed in the respective mutant in TnoGH1. All kinetic parameters of Hor GH1are summarised in Table 2.

\section{TnoGH1 and HorGH1 double mutant kinetic studies (pNT-Glc)}

164 All the TnoGH1 double mutants show a lower turnover rate with the target pNT-Glc compared to the wild type and, with the exception of the TnoGH1-V287R/H178Y, to the single mutants. In particular, TnoGH1166 L171K/V287R shows a 2-fold increase in specificity $\left(1820 \mathrm{M}^{-1} \mathrm{~s}^{-1}\right)$ but a 3-fold decrease in turnover rate. The 167 TnoGH1-L171K/ H178Y mutant shows a 3-fold decrease in specificity $\left(380 \mathrm{M}^{-1} \mathrm{~s}^{-1}\right)$ as well as a 10-fold decrease 168 in turnover rate. The TnoGH1-V287R/H178Y mutants shows a 2.5 -fold increase in specificity and a $1 / 3$-fold 169 decrease in turnover rate. TnoGH1-V287R/H178Y is the only variant in this series with a specificity $\left(2420 \mathrm{M}^{-1} \mathrm{~s}^{-}\right.$ $\left.170{ }^{1}\right)$ comparable to that observed in the single mutant TnoGH1-V287R $\left(2500 \mathrm{M}^{-1} \mathrm{~s}^{-1}\right)$.

171 With HorGH1 double mutants, the HorGH1-E173K/ M299R shows a 3-fold decrease in specificity and a 4172 fold decrease in turnover rate compared to the wild type (Table 2). The HorGH1-E173K/H180Y mutant shows 173 an 8-fold decrease in specificity and an 8-fold decrease in turnover. The HorGH1-M299R/H180Y mutant shows 174 a near 3-fold decrease specificity and a 4-fold decrease in turnover rate, similar to that seen in HorGH1-E173K/ 175 M299R.

176 As with single mutants, the pattern observed in TnoGH1 double mutants is closely mapped in the HorGH1 variants. All double mutants show a lower turnover rate compared to that of the single mutants or wild type.

178 Double mutants containing the arginine mutation (TnoGH1-V287R and HorGH1-M299R) show an increase in specificity. Kinetic parameters summarised in Table 1 and 2. 
The TnoGH1-V287R mutant shows a significant shift in specificity towards pNT-Glc which corresponds to $30 \%$ loss in specificity towards pNP-Glc without any loss in turnover rate (Table 1). In the analogous HorGH1 mutant, this observation is pronounced, as there is a 35 -fold loss in specificity towards the O-glycosidic substrate, as well as a 2-fold decrease in turnover number (Table 2). When comparing substrates, kinetic parameters for both enzymes appear to diverge to some extent in this case.

With pNT-Glc, TnoGH1-L171K shows a retention of kinetic parameters however with pNP-Glc, there is a 2.5-fold loss in specificity and a retention of turnover (Table 1). In HorGH1-E173K a decrease in kinetic parameters is observed with pNT-Glc, with pNP-Glc this is more pronounced, a 10-fold decrease in specificity is observed with a 2-fold decrease in turnover rate (Table 2). This mutation results in little perturbation in turnover rate for both substrates in TnoGH1, however a decrease in parameters is observed for both substrates in Hor GH1 (Table 1).

TnoGH1-H178Y mutant shows the lowest single mutant turnover rate with pNP-Glc, representing a 2-fold loss (Table 1). Similarly, Hor GH1-H180Y also shows a 2-fold decrease in specificity and turnover rate with the same substrate (Table 2). Kinetics parameters change in the same direction for both substrates in both enzymes on mutation at this position (Table 1 and Table 2).

Double mutants also show a complex relationship with respect to the native substrate. TnoGH1-L171K/V287R shows a 3 -fold loss in specificity and a 1/3-loss in turnover rate (Table 1) compared to the wild type. Whereas with pNT-Glc, this variant shows an increase in specificity (Table 1). The TnoGH1-L171K/H178Y mutant shows similar result with pNP-Glc as with pNT-Glc compared to the wild type, a 3-fold loss in specificity is observed in both specificity and turnover rate for pNP-Glc (Table 1). TnoGH1-V287R/H178Y shows a similar specificity towards pNP-Glc as the wild type enzyme, but a $1 / 3$ - loss in turnover rate compared to wild type, while with pNT-Glc a gain in specificity is observed compared to the wild type. (Table 1).

When $\operatorname{Hor} \mathrm{GH} 1$ double mutants are compared to the wild type with pNP-Glc substrate, all mutants show a loss in specificity and turnover rate. This is similar to the pattern observed with these mutants and the pNT-Glc. Unlike TnoGH1, the double mutants on Hor GH1 show the same change in kinetic parameters for both substrates. (Table 2).

\section{DISCUSSION}

208 Significantly enhanced activity towards a thiosaccharide substrate, pNT-Glc, was introduced in TnoGH1 and 209 Hor GH1 through rational design. The TnoGH1-V287R and HorGH1-M299R variants yielded the greatest 210 increase in specificity towards pNT-Glc while retaining similar turnover number to the wildtype. It has been 211 shown previously that R312 play a critical role in aphid myrosinase for sulfur recognition (Jones et al. 2002). In $212 \beta$-glycosidases, the amino acids at the equivalent position are hydrophobic in nature (valine in Tno and methionine 213 in Hor). A mutation to arginine introduces a guanidinium side chain in the active site capable of hydrogen bonding 214 to the thioglycosidic bond in the substrate. R312 may have a potential interaction that may stabilize the transition 215 state resulting in the observed increase in $k_{\text {cat }} / K_{\mathrm{M}}$. TnoGH1-L171K and Hor GH1-E173K, it also adds a positively 216 charged side chain into the active site, however an increase in the $k_{\text {cat }} K_{\mathrm{M}}$ is not observed. The crystal structure of 
217 BbMYR indicates K173 pointing away from the active site, possibly reducing its direct involvement in substrate

218 binding.

219 The introduction of a tyrosine in both TnoGH1 and HorGH1 to replace a histidine results in a dramatic decrease

220 in $k_{\text {cat }} / K_{\mathrm{M}}$ towards both pNP-Glc and pNT-Glc substrates compared to the wild types. Y180 (BbMYR numbering)

221 had been suggested to have a possible catalytic role due to the proximity of the sidechain to the thiosidic linkage

222 in glucosinolates. However, in both extremophilic enzymes, the histidine displayed at that position appears to be

223 highly conserved among $\beta$-glycosidases (Fig. 5), suggesting that a mutation at this position is poorly tolerated.

224 Specifically, tyrosine while not forbidden, has an incidence of less than $3 \%$ in the data set. The others two targeted

225 positions (L171 and V287, TnoGH1 numbering) are not as highly conserved (Fig. S9)

A more complex relationship is observed when the double mutants are compared to the wild type and single mutants. From the single mutant results, we observe a correlation between the introduction of polar residues in the active site of TnoGH1 and the specificity for the pNT-Glc substrate. This is supported by the change in specificity observed between the TnoGH1-H178Y mutant and the TnoGH1-V287R/H178Y double mutant, where the latter has a much-improved specificity when compared to the former. Likewise, when TnoGH1-V287R is combined with TnoGH1-L171K, the double mutant enzyme has a higher specificity than the TnoGH1-L171K but lower specificity than the TnoGH1-V287R variant.

We can also see that a similar effect was observed in HorGH1 specificity of pNT-Glc substrate with the HorGH1-M299R/H180Y double mutant. Unlike TnoGH1, double mutant HorGH1-E173K/M299R shows similar specificity to the HorGH1-E173K single mutant. We see a larger $K_{\mathrm{M}}$ (pNT-Glc) in the double mutant when compared to the HorGH1-M299R single mutant, and a large reduction in $k_{\text {cat }}$ compared to both single mutants. This would suggest that the same effect in the TnoGH1 mutants may be playing a role in the HorGH1 mutants.

The Hor GH1 and TnoGH1 mutants all exhibited a decrease in turnover rate and specificity with the native substrate pNP-Glc (Table 1 and 2). This indicates that the increase in specificity for pNT-Glc is at the expense of the native substrate. The increase observed in $k_{\mathrm{cat}} / K_{\mathrm{M}}$ (pNT-Glc) induced by the introduction of the arginine residues, is not observed with pNP-Glc. Considering the catalytic efficiency of the HorGH1-M299R, this mutant has a ratio of $k_{\text {cat }} / K_{\mathrm{M}}$ of glycoside to thioglycoside of 3:1, compared to the Hor $\mathrm{GH} 1$ wild type which has a ratio of 11:1.

244 In this study, enhanced $\beta$-thioglycosidase activity was introduced by rational design in the extremophilic $\beta$ glycosidases TnoGH1 and Hor GH1 by in-silico modelling of the B. Brassicae myrosinase. A three-fold increase in specificity for the thioglycosidic substrate with no loss in turnover number was observed by replacing of hydrophobic residues of both enzymes by arginine. These mutants were seen to have the greatest increase in specificity of all assayed mutants, including double mutants. Among the novel $\beta$-thioglycosidases addressed in this study, HorGH1-M299R is the most promising mutant for the industrial application due to the larger turnover number.

252 FP conceived and designed research. NA conducted experiments. FP, NA, and NRM analysed data. NA and NRM

253 drafted the manuscript and all authors read and approved the manuscript 
255 The authors thank the King Faisal University in Saudi Arabia for financial support (NA). NRM and FP were

256 funded by EraNet SUSFOOD2 (Project ID 122 ImPrOVE, through DEFRA UK) and by the Biotechnology and

257 Biological Research Council [grant number BB/P002536/1].

\section{Conflict of interest}

259 The authors declare that they have no conflict of interest.

\section{Ethical standards}

261 The authors conducted the research to the high ethical standards of the journal of submission.

\section{REFERENCES}

263 Babonneau D (2010) FitGISAXS: software package for modelling and analysis of GISAXS data using IGOR

264 Pro. J Appl Cryst 43(4):929-936. doi:10.1107/S0021889810020352

265

266 Bourderioux A, Lefoix M, Gueyrard D, Tatibouet A, Cottaz S, Arzt S, Burmeister WP, Rollin P (2005) The

267 glucosinolate-myrosinase system. New insights into enzyme-substrate interactions by use of simplified

268 inhibitors. Org Biomol Chem. 3(10):1872-1879. doi:10.1039/b502990b

269

270 Burmeister WP, Cottaz S, Driguez H, Iori R, Palmieri S, Henrissat B (1997) The crystal structures of Sinapis

271 alba myrosinase and a covalent glycosyl-enzyme intermediate provide insights into the substrate recognition

272 and active-site machinery of an S-glycosidase. Phytochemistry 5(5):663-676. doi:10.1016/S0969-

$273 \quad 2126(97) 00221-9$

274

275 Chen, C., Natale, D.A., Finn, R.D., Huang, H., Zhang, J., Wu, C.H. and Mazumder, R (2011) Representative

276 proteomes: a stable, scalable and unbiased proteome set for sequence analysis and functional annotation. Plos

277 One 6(4). doi:10.1371/journal.pone.0018910

278

279 Crooks GE, Hon G, Chandonia J-M, Brenner SE (2004) WebLogo: A Sequence Logo Generator.

280 Genome Res 14(6). doi:10.1101/gr.849004

281 Davies G, Henrissat B (1995) Structures and mechanisms of glycosyl hydrolases. Structure 3:853-859.

282 doi:10.1016/S0969-2126(01)00220-9

283 Dufour V, Stahl M, Baysse C (2015) The antibacterial properties of isothiocyanates. Microbiology 161(2):229-

284 243. doi:10.1099/mic.0.082362-0

285 El-Gebali S, Mistry J, Batman A, Eddy SR, Luciani A, Potter SC, Qureshi M, Richardson LJ, Salazar GA, Smart

286 A, Sonnhammer ELL, Hirsh L, Paladin L, Piovesan D, Tosatto SCE, Finn RD (2019) The Pfam protein families 287 database in 2019. Nucleic Acid Res 47:D427-D432. doi:10.1093/nar/gky995 
288 Gasteiger E, Hoogland C, Gattiker A, Duvaud Sv, Wilkins MR, Appel RD, Bairoch A (2005) Protein

289 Identification and Analysis Tools on the ExPASy Server. in The proteomics protocols handbook 571-607. doi:

$290 \quad 10.1385 / 1-59259-890-0: 571$

291 Halkier BA, Gershenzon J (2006) Biology and Biochemistry of Glucosinolate. Annu Rev Plant Biol 57:303-333.

292 doi:10.1146/annurev.arplant.57.032905.105228

293 He XY, Wang XQ, Yang SJ, Chang WR, Liang DC (2001) Overexpression, purification, crystallization and

294 preliminary crystallographic studies on a thermostable $\beta$-glycosidase from Thermus nonproteolyticus HG102.

295 Acta Crystallogr D 57(11):1650-1651. doi:10.1107/s090744490101112x

296

297 Heckmann CM (2017) Construction of Expression Vectors for Selective His-tag Removal. University of 298 Nottingham

299 Henn-Sax M, Höcker B, Wilmanns M, Sterner R (2001) Divergent evolution of (betaalpha)8-barrel enzymes. Biol 300 Chem 382(9):1315-20. doi:10.1515/BC.2001.163

301 Husebye H, Arzt S, Burmeisterc WP, Frauke V. Hartelf, Brandtf A, Rossiterg JT, Bonesa AM (2005) Crystal 302 structure at $1.1 \AA$ resolution of an insect myrosinase from Brevicoryne brassicae shows its close relationship to $303 \beta$-glucosidases. Insect Biochem Molec 35(12):1311-1320. doi:10.1016/j.ibmb.2005.07.004

304 Jones AME, Winge P, Bones AM, Cole R, Rossiter JT (2002) Characterization and evolution of a myrosinase 305 from the cabbage aphid Brevicoryne brassicae. Insect Biochem Molec 32(3):275-284. doi:10.1016/S0965306 1748(01)00088-1

307 Kori LD, Hofmannb A, Patela BKC (2011) Expression, purification and preliminary crystallographic analysis of 308 the recombinant $\beta$-glucosidase (BglA) from the halothermophile Halothermothrix orenii. Acta Crystallogr F

309 67(1):111-113. doi:10.1107/S1744309110046981

310

311 Kumar S, Stecher G, Li M, Knyaz C, Tamura K (2018) MEGA X: Moleculr Evolutionary Genetics Analysis 312 across Computing Platform. Mol Biol Evol 35(6) 1547-1549. doi:10.1093/molbev/msy096

313 Nong H, Zhang JM, Li DQ, Wang M, Sun XP, Zhu YJ, Meijer J, Wang QH (2010) Characterization of a novel $\beta$ 314 thioglucosidase CpTGG1 in Carica papaya and its substrate-dependent and ascorbic acid-independent O- $\beta$ 315 glucosidase activity. J Integr 52(10):879-890. doi:10.1111/j.1744-7909.2010.00988.x

316 Park S, Lee B, Park K (2017) Extremophilic Carbohydrate Active Enzymes (CAZymes). J Nutr Health Food 317 Eng 7(1):1-9. doi:10.15406/jnhfe.2017.07.00230

318 Pettersen EF, Goddard TD, Huang CC, Couch GS, Greenblatt DM, Meng EC, Ferrin TE (2004) UCSF

319 Chimera-A Visualization System for Exploratory Research and Analysis. J Comput Chem 25(13):1605-1612.

320 doi:10.1002/jcc.20084 
321 Pontoppidan B, Ekbom B, Eriksson S, Meijer J (2001) Purification and characterization of myrosinase from the

322 cabbage aphid (Brevicoryne brassicae), a brassica herbivore. Euro J Biochem 268(4):1041-1048.

323 doi:10.1046/j.1432-1327.2001.01971.x

324 Rakariyatham N, Butrindr B, Niamsup H, Shank L (2005) Screening of filamentous fungi for production of 325 myrosinase. Braz J Microbiol 36(3): 242-245. doi:10.1590/S1517-83822005000300007

326 Rice P, Longden I, Bleasby A (2000) EMBOSS: the European molecular biology open software suite.

327 Trends Biochem Sci 16(6):276-277. doi:10.1016/s0168-9525(00)02024-2

328

329 Robert X, Gouet P (2014) Deciphering key features in protein structures with the new ENDscript server. Nucleic 330 Acids Res 42(W1):W320-W324. doi:10.1093/nar/gku316

331 Samec D, Pavlovic' I, Salopek-Sondi B (2017) White cabbage (Brassica oleracea var. capitata f. alba): botanical, 332 phytochemical and pharmacological overview. Phytochem Rev 16(1):117-135. doi:10.1007/s11101-016-9454-4

333 Silverman JA, Balakrishnan R, Harbury PB (2001) Reverse engineering the $(\beta / \alpha) 8$ barrel fold. Proc Natl Acad

334 Sci 98(6):3092-3097. doi:10.1073/pnas.041613598

335 Swift ML (1997) GraphPad Prism, Data Analysis, and Scientific Graphing. J Chem Inf Model 37(2):411-412.

336 doi:10.1021/ci960402j

337

338 Wang X, He X, Yang S, An X, Chang W, Liang D (2003) Structural Basis for Thermostability of $\beta$ -

339 Glycosidase from the Thermophilic Eubacterium Thermus nonproteolyticus HG102. J Bacteriol Res

340 185(14):4248-4255. doi:10.1128/JB.185.14.4248-4255.2003

341

342 Winde I, Wittstock U (2011) Insect herbivore counteradaptations to the plant glucosinolate-myrosinase system.

343 Phytochemistry 72(13):1566-1575. doi:10.1016/j.phytochem.2011.01.016

344 Witzel K, Hanschen FS, Klopsch R, Ruppel S, Schreiner M, Grosch R (2015) Verticillium longisporum

345 infection induces organ-specific glucosinolate degradation in Arabidopsis thaliana.

346 Front Plant Sci 6: 508. doi:10.3389/fpls.2015.00508

347

348 Yin J, Chen JC, Wu Q, Chen GQ (2015) Halophiles, coming stars for industrial biotechnology. Biotechnol Adv 349 33(7):1433-1442. doi:10.1016/j.biotechadv.2014.10.008

350 
352 Fig. 1. Protein database structures of the active sites of glycoside enzymes. a Thermus nonproteolyticus glycoside 353 hydrolase (pdb:1NP2); the residues mutated in this study are indicated, L171, H178 and V287. Residues essential 354 for activity also indicated, E164 and E338. b Halothermothrix orenii glycoside hydrolase (pdb:3TA9); the residues mutated in this study are indicated, E173, H180 and M299. Residues essential for activity also indicated,

356 E166 and E354. c diagram indicating the relationship between the substrate and the side chains of the residues of 357 wild type Thermus nonproteolyticus mutated in this study, essential residues also displayed. Distances between 358 the glycosidic substrate and sidechains predicted with UCSF Chimera. d diagram indicating the relationship 359 between the glycosidic substrate and the side chains of the residues of wild type Halothermothrix orenii mutated 360 in this study, essential residues also displayed. Distances between substrate and sidechains predicted with UCSF 361 Chimera.

362 Fig. 2. Protein database structures of the active sites of myrosinase enzymes. a S. alba myrosinases (pdb:1E4M); 363 residues essential for activity also indicated, Q187 and E409. b B. brassicae myrosinases (pdb:1WCG); target 364 residues of mutagenesis indicated, K173, Y180 and R312; residues essential for activity also indicated, E167 and 365 E374. c diagram indicating the relationship between the thioglycosidic substrate and the side chains of the residues 366 of wild type B. brassicae myrosinases targeted residues of mutagenesis, essential residues also displayed. 367 Distances between substrate and sidechains predicted with UCSF Chimera.

368 Fig. 3. Amino acid sequence alignment of Thermus nonproteolyticus glycoside hydrolase (T. nonproteolyticus), 369 Halothermothrix orenii glycoside hydrolase (H. orenii), B. brassicae myrosinases (B. brassicae), and S. alba 370 myrosinases (S. alba).

371 Fig. 4. Structures of the substrates, the native substrate of myrosinase is added to show the similarity in the 372 structure of the molecules. a 4-nitrophenyl $\beta$-D-thioglucopyranoside (pNT-Glc). b 4-nitrophenyl- $\beta$-D373 Glucopyranoside (pNP-Glc). c sinigrin, the native substrate of myrosinase.

374 Fig. 5 Sequence logo generated for amino acid position 178 (Thermus nonproteolyticus glycoside hydrolase 375 numbering) of glycosyl hydrolase family 1, indicating that cystine and histidine are the most highly conserved at 376 position 178. Sequences were taken from Pfam, the representative proteome at 15\% co-membership threshold 377 (approximately 3900 sequences) was aligned with the wild type sequences of the $\beta$-glycosidase used in this study. 378 Sequence logo was generated with WebLogo. 
379 Table 1: Table summarizing the kinetic parameters of the wild type enzyme and mutants of Thermus 380 nonproteolyticus (TnoGH1) against the 4-nitrophenyl $\beta$-D-thioglucopyranoside (pNT-Glc) and 4-nitrophenyl- $\beta$ 381 -D-Glucopyranoside (pNP-Glc). Experiments were conducted in triplicate. Standard errors are given, based on 382 fitted theoretical curves.

\begin{tabular}{|c|c|c|c|c|c|c|}
\hline & $\begin{array}{l}k_{\text {cat }} / K_{\mathrm{M}}(\mathrm{pNT}- \\
\mathrm{Glc}) / \mathrm{M}^{-1} \mathrm{~s}^{-1}\end{array}$ & $\begin{array}{l}k_{\text {cat }}(\mathrm{pNT}- \\
\mathrm{Glc}) / \mathrm{s}^{-1}\end{array}$ & $\begin{array}{c}K_{\mathrm{M}}(\mathrm{pNT}-\mathrm{Glc}) / \\
\mathrm{mM}\end{array}$ & $\begin{array}{c}k_{\text {cat }} / K_{\mathrm{M}}(\mathrm{pNP}-\mathrm{Glc}) / \\
\mathrm{M}^{-1} \mathrm{~s}^{-1}\end{array}$ & $\begin{array}{l}k_{\text {cat }}(\mathrm{pNP}- \\
\mathrm{Glc}) / \mathrm{s}^{-1}\end{array}$ & $\begin{array}{c}K_{\mathrm{M}}(\mathrm{pNP}-\mathrm{Glc}) / \\
\mathrm{mM}\end{array}$ \\
\hline WT TnoGH1 & $940 \pm 70$ & $1.34 \pm 0.04$ & $1.40 \pm 0.10$ & $\begin{array}{c}246 \times 10^{3} \pm 3 \times \\
10^{3}\end{array}$ & $132 \pm 3$ & $0.54 \pm 0.06$ \\
\hline TnoGH1-L171K & $1200 \pm 100$ & $1.10 \pm 0.04$ & $0.90 \pm 0.10$ & $92 \times 10^{3} \pm 9 \times 10^{3}$ & $136 \pm 4$ & $1.50 \pm 0.20$ \\
\hline TnoGH1-V287R & $2500 \pm 400$ & $1.39 \pm 0.01$ & $0.56 \pm 0.01$ & $\begin{array}{c}190 \times 10^{3} \pm 20 \times \\
10^{3}\end{array}$ & $117 \pm 3$ & $0.61 \pm 0.07$ \\
\hline TnoGH1-H178Y & $300 \pm 50$ & $0.15 \pm 0.01$ & $0.27 \pm 0.07$ & $\begin{array}{c}230 \times 10^{3} \pm 36 \times \\
10^{3}\end{array}$ & $77 \pm 2$ & $0.34 \pm 0.06$ \\
\hline $\begin{array}{c}\text { TnoGH1- } \\
\text { L171K/V287R }\end{array}$ & $1820 \pm 320$ & $0.08 \pm 0.01$ & $0.21 \pm 0.04$ & $58 \times 10^{3} \pm 5 \times 10^{3}$ & $116 \pm 3$ & $1.90 \pm 0.20$ \\
\hline $\begin{array}{c}\text { TnoGH1- } \\
\text { L171K/H178Y }\end{array}$ & $380 \pm 80$ & $0.09 \pm 0.04$ & $0.22 \pm 0.05$ & $\begin{array}{c}110 \times 10^{3} \pm 10 \times \\
10^{3}\end{array}$ & $69 \pm 2$ & $0.70 \pm 0.10$ \\
\hline $\begin{array}{c}\text { TnoGH1- } \\
\text { V287R/H178Y }\end{array}$ & $2420 \pm 500$ & $0.84 \pm 0.04$ & $0.35 \pm 0.08$ & $\begin{array}{c}260 \times 10^{3} \pm 40 \times \\
10^{3}\end{array}$ & $106 \pm 3$ & $0.41 \pm 0.07$ \\
\hline
\end{tabular}


384 Table 2: Table summarizing the kinetic parameters of the wild type enzyme and mutants of Halothermothrix 385 orenii (Hor GH1) against the 4-nitrophenyl $\beta$-D-thioglucopyranoside (pNT-Glc) and 4-nitrophenyl- $\beta$-D386 glucopyranoside (pNP-Glc). Experiments were conducted in triplicate. Standard errors are given, based on fitted 387 theoretical curves.

\begin{tabular}{|c|c|c|c|c|c|c|}
\hline & $\begin{array}{c}k_{\mathrm{cat}} / K_{\mathrm{M}}(\mathrm{pNT}-\mathrm{Glc}) \\
/ \mathrm{M}^{-1} \mathrm{~s}^{-1}\end{array}$ & $\begin{array}{c}k_{\text {cat }}(\mathrm{pNT}-\mathrm{Glc}) / \\
\mathrm{s}^{-1}\end{array}$ & $\begin{array}{c}K_{\mathrm{M}}(\mathrm{pNT}-\mathrm{Glc}) / \\
\mathrm{mM}\end{array}$ & $\begin{array}{l}k_{\text {cat }} / K_{\mathrm{M}}(\mathrm{pNP}- \\
\mathrm{Glc}) / \mathrm{M}^{-1} \mathrm{~s}^{-1}\end{array}$ & $\begin{array}{c}k_{\mathrm{cat}}(\mathrm{pNP}-\mathrm{Glc}) / \\
\mathrm{s}^{-1}\end{array}$ & $\begin{array}{c}K_{\mathrm{M}}(\mathrm{pNP}-\mathrm{Glc}) / \\
\mathrm{mM}\end{array}$ \\
\hline WT HorGH1 & $9083 \pm 529$ & $37.2 \pm 0.7$ & $4.1 \pm 0.3$ & $102000 \pm 7 \times 10^{3}$ & $80 \pm 1$ & $0.5 \pm 0.1$ \\
\hline HorGH1-E173K & $3331 \pm 153$ & $19.1 \pm 0.3$ & $5.7 \pm 0.3$ & $11000 \pm 1 \times 10^{3}$ & $26 \pm 2$ & $2.5 \pm 0.5$ \\
\hline HorGH1-M299R & $13260 \pm 170$ & $33.7 \pm 1.2$ & $2.5 \pm 0.4$ & $36000 \pm 2 \times 10^{3}$ & $34 \pm 1$ & $0.9 \pm 0.1$ \\
\hline HorGH1-H180Y & $2501 \pm 131$ & $9.4 \pm 0.2$ & $3.8 \pm 0.2$ & $55000 \pm 5 \times 10^{3}$ & $38 \pm 1$ & $0.7 \pm 0.1$ \\
\hline $\begin{array}{c}\text { HorGH1- } \\
\text { E173K/M299R }\end{array}$ & $2622 \pm 122$ & $10.1 \pm 0.2$ & $3.8 \pm 0.2$ & $7000 \pm 1 \times 10^{3}$ & $12 \pm 1$ & $1.6 \pm 0.1$ \\
\hline $\begin{array}{c}\text { HorGH1- } \\
\text { E173K/H180Y }\end{array}$ & $1080 \pm 61$ & $5.2 \pm 0.1$ & $4.8 \pm 0.3$ & $15000 \pm 1 \times 10^{3}$ & $24 \pm 1$ & $1.8 \pm 0.2$ \\
\hline $\begin{array}{c}\text { HorGH1- } \\
\text { M299R/H180Y }\end{array}$ & $3770 \pm 198$ & $7.3 \pm 0.1$ & $1.9 \pm 0.1$ & $23000 \pm 3 \times 10^{3}$ & $25 \pm 2$ & $1.1 \pm 0.2$ \\
\hline
\end{tabular}

